\title{
Low Dose Capecitabine as Maintenance Therapy in Colorectal Cancer with Irresectable Metastasis
}

\author{
Atef Yousef ${ }^{1}$, Lobna Ezz EIArab ${ }^{1}$, Ahmed Ezzat ${ }^{1}$, Ghada Refaat ${ }^{1}$ \\ Clinical Oncology and Nuclear Medicine Department, Ain Shams University, Cairo, Egypt
}

Background and aim: In metastatic colorectal cancer (mCRC), there is now a desire to prolong overall survival (OS) by using individualized therapeutic strategy and minimal toxicity. This study was conducted to assess the efficacy and safety of the use of maintenance low dose capecitabine, an oral fluoropyrimidine carbamate that proved efficacy in treatment of advanced colorectal cancer, after best response achieved with previous standard chemotherapy.

Methods: This is a prospective study, conducted on 40 colorectal cancer patients with irresectable metastasis, after achieving objective response with standard chemotherapy. Capecitabine was given at a dose of 500 $\mathrm{mg} / \mathrm{m}^{2}$ (maximum $1000 \mathrm{mg}$ total) twice daily for 5 days/week continuously till progression or unacceptable toxicity. Evaluation was done every 3 cycles or earlier in case of suspected progression. Adverse events, progression free survival (PFS), and survival follow-up data were collected.

Results: The study regimen proved to be quite tolerable. Main toxicities were hand-foot syndrome $(60 \%)$ and fatigue (50\%). Only 7.5\% developed grade III hand-foot syndrome. The median time to progression was 34 weeks. Overall survival was $65.2 \%$ and $59.8 \%$ at one and two years respectively. Synchronous metastasis had a significant negative impact on PFS in comparison to metachronous metastases. Better performance status and good objective response to previous chemotherapy had significant positive impact on both PFS and OS. Conclusion: The results suggest that low dose capecitabine is effective in maintaining response in mCRC with good tolerability. Further exploration in larger prospective studies is needed.

Key words: Metastatic Colorectal Cancer, Capecitabine, Maintenance Therapy

Corresponding Author: Ghada Refaat_E-mail: ghada_refaat@med.asu.edu.eg

\section{INTRODUCTION}

Advances have been made over the past decade in the treatment of metastatic colorectal cancer (mCRC), where there is significant increase in response rate as well as increase in the median survival from 5 months to 2 years, as a result of advances in surgical techniques, newer options in chemotherapy and the emergence of effective biotherapy ${ }^{1}$.Given patients' preferences for more tolerable regimens and improved quality of life, treatment approaches have changed from continuous high-dose aggressive therapy until disease progression to either chemotherapy-free intervals or reduced-dose, lesstoxic maintenance regimens $\mathrm{s}^{2,3}$.

Maintenance therapy refers to the close, regular administration of a chemotherapeutic drug at relatively low (non-toxic) doses, over prolonged periods, with no extended drug-free break periods ${ }^{4}$. The main targets of continuous chemotherapy are the endothelial cells of the growing vasculature of a tumor ${ }^{4}$. Maintenance therapy can be integrated and sequenced with standard maximum tolerated dose type chemotherapy where brief courses of such induction therapy, given 'upfront', is followed by long term maintenance low-dose chemotherapy, or combined with a concurrent targeted therapy, especially antiangiogenic drugs such as anti - vascular endothelial growth factor receptor (VEGFR) -2 antibodies ${ }^{5}$.

Among the advantages of maintenance chemotherapy is reduction in acute toxicities, hospital admission costs and increased patient's convenience. This can be done by using oral drugs that can be taken at home ${ }^{6}$.

Several trials are being performed to evaluate the efficacy, safety and quality of life through the use of maintenance therapy in advanced colorectal cancer, using many agents like irinotecan ${ }^{7}$, infusion fluorouracil $(5-\mathrm{FU})^{8}$ and oral fluoropyrimidines ${ }^{9-15}$, as well as targeted therapy like bevacizumab and erlotinib ${ }^{32-36}$.

Capecitabine is an oral 5-FU prodrug that is modified via a different metabolic pathway than other oral 5-FU derivatives. Compared to intravenous 5-FU, capecitabine is associated with a lower incidence and severity of diarrhea, stomatitis, nausea, and neutropenia but an 


$\begin{array}{llll}\text { Vol. } 11 \text { No. } 1-2 & 2015 & \text { Yousef A. et al }\end{array}$

increased rate of hand-foot syndrome (HFS) $)^{12}$. Its high concentration in tumor tissue increases both the efficacy and tolerability of the agent through targeted delivery. Its oral administration simplifies care, frequently precluding the need for central venous access or infusion pumps ${ }^{16}$.

Compared to other oral 5-FU derivatives such as S-1 and tegafur-uracil, capecitabine is approved by the United States Food and Drug Administration (FDA) and has been extensively studied in gastrointestinal malignancies. Capecitabine has been shown to be a safe and efficacious alternative to bolus 5-FU for mCRC in phase III clinical trials ${ }^{17,18}$.

Several clinical trials are currently under way which will help to further elucidate the role of chemo-holidays with periods of low dose maintenance therapy on patient outcome and quality of life in the era of the routine use of conventional chemotherapy plus targeted agents as first-line standard of care.

The aim of this study was to assess the efficacy and safety of the use of maintenance low dose capecitabine, after best response achieved with previous standard chemotherapy in mCRC patients with irresectable metastasis.

\section{PATIENTS AND METHODS}

\section{Study design}

This single-arm, phase II study was conducted in the Clinical Oncology department, Ain Shams University hospitals in the period from July 2009 to December 2012. The protocol was reviewed and approved by the Research Ethical Committee (REC) at the Faculty of Medicine, Ain Shams University on $28^{\text {th }}$ June, 2009 (FMASU 234/ 2009).

\section{Patient population}

Inclusion criteria included irresectable mCRC with expected survival of more than 3 months. The age included is above 18 years with Eastern Cooperative Oncology Group (ECOG) performance status of $0-2,{ }^{19}$ and adequate bone marrow, renal and liver functions. Patients had to achieve objective response according to the Response Evaluation Criteria in Solid Tumors (RECIST) criteria $^{20}$ after one or more of standard chemotherapy regimens for metastatic disease with at least one bidimensionally measurable lesion by computerized tomography (CT) and/or magnetic resonance imaging (MRI) at the initiation of standard chemotherapy. The interval between completion of the former chemotherapy regimen and starting capecitabine should not exceed 4 weeks.
Exclusion criteria included tumor progression before recruitment, ascites or pleural effusions as the only assessable lesions, planned radical resection of metastatic disease and symptomatic cerebral metastases. Any other concurrent severe or uncontrolled diseases that may significantly impair the absorption or affect the tolerance of the oral drug were excluded. These included history of dihydropyrimidine dehydrogenase deficiency (DPD), uncontrolled gastrointestinal disease (e.g nausea, vomiting, diarrhea, malabsorption syndrome, or bowel obstruction) and pregnancy.

\section{Pretreatment evaluation}

Medical history, clinical examination, complete blood picture, complete liver and kidney function tests, baseline serum carcinoembryonic antigen (CEA) level, electrocardiography (ECG), CT scan and/or MRI (baseline evaluation according to sites of metastases).

\section{Treatment plan}

Capecitabine was given at a dose of $500 \mathrm{mg} / \mathrm{m}^{2}$ (with a maximum of $1000 \mathrm{mg}$ total dose) twice daily for 5 days/week, administered orally one hour after breakfast and evening meals. The dose was calculated according to body surface area, however, the majority of patients had received the maximum dose of $1000 \mathrm{mg}$ twice daily (2 Tablets bid). It was given continuously till either disease progression or unacceptable toxicity.

In the event of patients developing drug-related grade 3 adverse events, laboratory abnormalities, or in patients in whom the investigator judged continuation of treatment unfeasible, treatment was delayed for up to 4 weeks to allow patient recovery.

When continuation of treatment at the same dose considered being intolerable due to adverse events, irrespective of grade, the drug was administered with $25 \%$ dose reduction.

Treatment was permanently discontinued in case of persistent drug intolerability, even after dose reduction and transient discontinuation.

\section{Study assessment}

One treatment cycle was defined as a 4-week treatment. Complete blood counts were repeated every 2 weeks, and biochemical tests were done every 4 weeks.

Symptoms were followed and regular clinical staging was performed every 3 cycles using CT scan and/or MRI and serum CEA level. Evaluation was done earlier in the case of clinical progression. 
Adverse events were evaluated using the National Cancer Institute Common Terminology Criteria for Adverse Events - version 3 (CTCAE v3.0) ${ }^{21}$ every cycle.

\section{Evaluation of efficacy}

Maintenance of the tumor response was evaluated every 3 cycles or earlier in case of suspected progression. Response of previous chemotherapy as well as current study treatment was measured according to RECIST criteria. ${ }^{20}$ Progression free survival (PFS) was calculated from the date of study entry until objective tumor progression or death. Overall survival (OS) was calculated from the date of study entry until the last follow up visit or death.

\section{Evaluation of safety}

Safety was evaluated in all patients who received Capecitabine treatment, and adverse event frequencies were recorded and graded in accordance with the CTCAE v3.0. ${ }^{21}$. HFS (or palmar-plantar erythrodysesthesia) was classified as grade 1, painless mild skin changes; grade 2, pain or skin changes including peeling, blisters, bleeding, edema not affecting daily function; grade 3, painful skin changes affecting daily function ${ }^{22}$.

\section{Statistical analysis}

Kaplan-Meier Survival Analysis was used to examine the distribution of time-to-event variables and to compare the distribution by levels of a factor variable or produce separate analyses by levels of a stratification variable. Log rank test was used to compare time-toevent variables by levels of a factor variable.

\section{RESULTS}

\section{Patients charecteristics}

Patients' baseline characteristics are shown in (Table 1) Forty patients were recruited, with median age of 51 years ranging from 29 to 75 years (24 female and 16 male), ECOG performance status from 0 to 2, all patients had colorectal cancer (29 colon, 11 rectal) with unresectable metastases (synchronous metastases in 27 patients and metachronous metastases in 13 patients) where 29 patients had 1 metastatic site, 10 had 2 and 1 had 3 metastatic sites.

Thirty one patients underwent previous surgery (24 to primary, 4 to secondary, 3 to both), 6 patients had received prior radiotherapy, and all patients had received one (15 patients) or more chemotherapy lines (25 patients) for metastatic disease. Response of previous chemotherapy (before the current study) was: $\mathrm{CR}$ in 10 patients $(25 \%), \mathrm{PR}$ in 2 patients $(5 \%)$ and $\mathrm{SD}$ in 28 patients $(70 \%)$.

\section{Treatment duration and outcome}

The median duration of treatment was 34 weeks with standard of error of 4.096 weeks (ranging from 4 to 86 weeks). The mean number of cycles received was 7.5 cycles \pm 5.1 as standard of deviation, ranging from 1 to 21.5 cycles. The median follow up interval was 12 months ranging from 1 to 30 months.

Out of 40 patients recruited in this study, 35 patients $(87.5 \%)$ developed disease progression, while the study was terminated in 2 patients $(5 \%)$ for toxicity, and 3 patients $(7.5 \%)$ for unrelated medical or surgical conditions.

By the end of the study, 26 patients (65\%) were still alive, while 14 patients (35\%) died.

\section{Safety}

Table 2 shows the frequency of adverse events. No grade 4 toxicities were detected during the use of low dose capecitabine. Grade III was detected in only 3 patients $(7.5 \%)$ who developed grade III HFS. Nonhematological toxicities in the current study included: HFS (60\%), fatigue (50\%), anorexia (32.5\%), abdominal pain $(31.5 \%)$, diarrhea $(22.5 \%)$, hyperbilirubinemia $(17.5 \%)$, mucositis $(17.5 \%)$, nausea $(5 \%)$, and vomiting (5\%). Hematological toxicities were neutropenia $(17.5 \%)$, anemia $(12.5 \%)$, and thrombocytopenia (5\%).

\section{Treatment compliance}

Only 3 patients had developed grade III HFS in the $3^{\text {rd }}, 4^{\text {th }}$, and $8^{\text {th }}$ cycle of treatment, where treatment was delayed for 1 to 3 weeks to allow patient recovery.

In 4 patients, when their full doses were intolerable due to G II-III HFS at $1^{\text {st }}, 7^{\text {th }}, 8^{\text {th }}$ and $9^{\text {th }}$ cycle, $25 \%$ dose reduction was applied. The dose was reduced from 2 tablets $(1000 \mathrm{mg})$ twice daily to 3 tablets daily in those 4 patients.

Treatment was permanently discontinued in 2 patients who developed persistent drug intolerability due to HFS, even after dose reduction and transient discontinuation. 


\section{Efficacy}

The median PFS was 34 weeks with standard of error of 4.096 weeks ranging from 4 to 86 weeks $(95 \% \mathrm{CI}$ : $26-42)$.

PFS differed significantly according to gender ( $p$ value $=0.021)$ being longer in females, a finding mostly related to greater number of females recruited in the study.

PFS differed significantly according to the performance status. ECOG PS significantly affected the mean PFS ( $p$ value $=0.019$ ). It was 56.7 weeks for PS 0 (95\% CI: 38.6 - 74.9), 27.7 weeks for PS 1 (95\% CI: 21.3 - 34) and 17.3 weeks for PS 2 (95\% CI: 0 - 34.8).

PFS was also significantly different according to the time to metastases ( $\mathrm{p}$ value $=0$.048). Mean PFS for synchronous metastases was 30.1 weeks (95\% CI: 21.7 38.4), while the mean PFS for metachronous metastases was 49 weeks (95\% CI: 30.2 - 67.8).

The response to previous chemotherapy was also associated with significant difference in PFS ( $p$ value= 0.05). Mean PFS for patients who had achieved CR or PR was 53.7 weeks (95\% CI: 33.5 - 73.9), while the

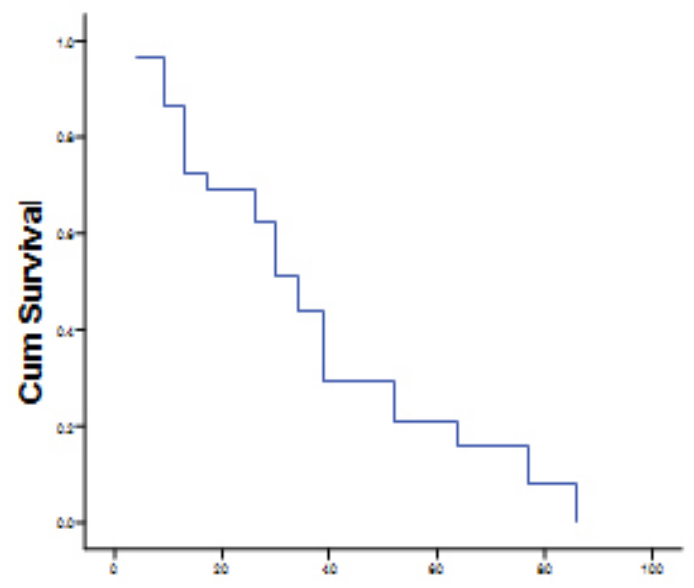

Figure 1: Progression free survival (in weeks)

Figure 1: Progression free survival (in weeks). mean PFS for patients who had achieved SD was 31.4 weeks (95\% CI: 21.8 - 41).

Difference in PFS was not significant between patients $\leq 50$ years of age or above, colon versus rectum as a primary site, number of metastatic sites, previous surgery to secondaries, or number of previous chemotherapy lines received.

One year survival was $65.2 \%$, and the estimated 2 years survival is $59.8 \%$, with significant difference between patients with different performance status $(p$ value $=0.021)$ and response achieved with previous chemotherapy $(p$ value $=0.021)$.

Kaplan-Meier curves for PFS and OS are shown in Figures 1 and 2, respectively.

\section{Further management after study termination}

After study termination, 23 patients $(57.5 \%)$ received standard palliative chemotherapy for metastatic disease, 1 patient $(2.5 \%)$ received palliative chemo-radiation (for recurrent rectal mass), 1 patient $(2.5 \%)$ underwent palliative surgery (for recurrent obstructing colonic mass), while 15 patients $(37.5 \%)$ have undergone no further management (best supportive care).

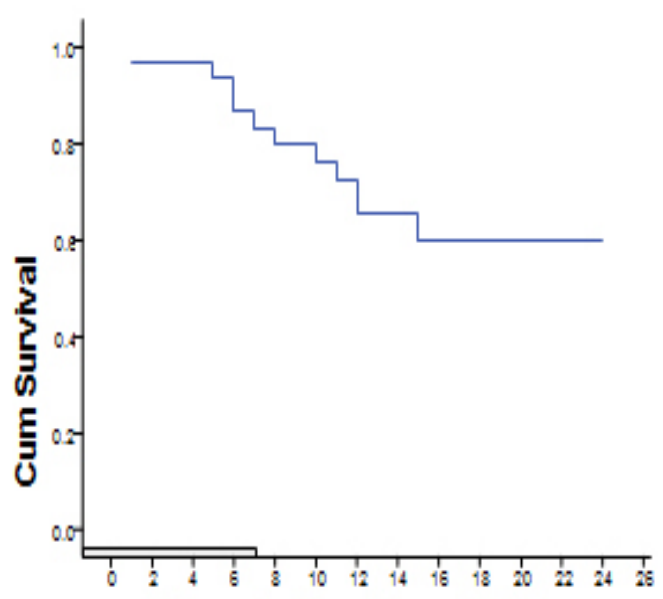

Figure 2: 0verall survival (in months)

Figure 2: Overall survival (in months). 
Table (1): Baseline characteristics of study cases

\begin{tabular}{|c|c|c|c|}
\hline & & $\mathbf{N}$ & $\%$ \\
\hline \multirow{2}{*}{ Age group } & $\leq 50$ Years & 18 & $45.0 \%$ \\
\hline & $>50$ Years & 22 & $55.0 \%$ \\
\hline \multirow{2}{*}{ Age } & Mean \pm SD & $51.2 \pm 13.2$ & \\
\hline & Range & $29-75$ & \\
\hline \multirow{2}{*}{ Gender } & Male & 16 & $40.0 \%$ \\
\hline & Female & 24 & $60.0 \%$ \\
\hline \multirow{2}{*}{ Primary site } & Colon & 29 & $72.5 \%$ \\
\hline & Rectum & 11 & $27.5 \%$ \\
\hline \multirow[t]{2}{*}{ Time to metastases } & Synchronous & 27 & $67.5 \%$ \\
\hline & Metachronous & 13 & $32.5 \%$ \\
\hline \multirow{3}{*}{ Number of metastases } & 1 & 29 & $72.5 \%$ \\
\hline & 2 & 10 & $25.0 \%$ \\
\hline & 3 & 1 & $2.5 \%$ \\
\hline \multirow{2}{*}{ Number of metastases } & Mean \pm SD & $1.28 \pm .45$ & \\
\hline & Range & $1-3$ & \\
\hline \multirow{13}{*}{ Sites of metastases } & Liver & 13 & $32.5 \%$ \\
\hline & Peritoneum & 7 & $17.5 \%$ \\
\hline & liver/lymph nodes & 4 & $10 \%$ \\
\hline & Abdominal mass & 2 & $5 \%$ \\
\hline & Pelvic mass & 2 & $5 \%$ \\
\hline & Peritoneum/lymph nodes & 2 & $5 \%$ \\
\hline & Peritoneum/uterus & 2 & $5 \%$ \\
\hline & Bladder & 1 & $2.5 \%$ \\
\hline & Liver/ bone & 1 & $2.5 \%$ \\
\hline & Liver/ lung & 1 & $2.5 \%$ \\
\hline & Lymph nodes & 1 & $2.5 \%$ \\
\hline & Lung & 1 & $2.5 \%$ \\
\hline & Uterus/ peritoneum/ lung & 1 & $2.5 \%$ \\
\hline \multirow{4}{*}{ Previous surgery } & No previous surgery & 9 & $22.5 \%$ \\
\hline & Previous surgery to primary & 24 & $60.0 \%$ \\
\hline & Previous surgery to secondary & 4 & $10.0 \%$ \\
\hline & Previous surgery to both & 3 & $7.5 \%$ \\
\hline \multirow{3}{*}{$\begin{array}{l}\text { Chemotherapy lines } \\
\text { for metastatic disease }\end{array}$} & One line & 15 & $37.5 \%$ \\
\hline & Two lines & 17 & $42.5 \%$ \\
\hline & Three lines & 8 & $20.0 \%$ \\
\hline
\end{tabular}


Table 2: Description of treatment toxicity among study cases

\begin{tabular}{|c|c|c|c|}
\hline & Grade & $\mathbf{N}$ & $\%$ \\
\hline \multirow[t]{4}{*}{ Hand-foot syndrome } & 0 & 16 & $40.0 \%$ \\
\hline & I & 16 & $40.0 \%$ \\
\hline & II & 5 & $12.5 \%$ \\
\hline & III & 3 & $7.5 \%$ \\
\hline \multirow[t]{3}{*}{ Fatigue } & 0 & 20 & $50.0 \%$ \\
\hline & I & 19 & $47.5 \%$ \\
\hline & II & 1 & $2.5 \%$ \\
\hline \multirow[t]{2}{*}{ Diarrhea } & 0 & 31 & $77.5 \%$ \\
\hline & I & 9 & $22.5 \%$ \\
\hline \multirow[t]{3}{*}{ Anorexia } & 0 & 27 & $67.5 \%$ \\
\hline & I & 12 & $30.0 \%$ \\
\hline & II & 1 & $2.5 \%$ \\
\hline \multirow[t]{3}{*}{ Abdominal pain } & 0 & 31 & $77.5 \%$ \\
\hline & I & 8 & $20.0 \%$ \\
\hline & II & 1 & $2.5 \%$ \\
\hline \multirow[t]{2}{*}{ Elevated bilirubin } & 0 & 33 & $82.5 \%$ \\
\hline & I & 7 & $17.5 \%$ \\
\hline \multirow[t]{2}{*}{ Mucositis } & 0 & 33 & $82.5 \%$ \\
\hline & I & 7 & $17.5 \%$ \\
\hline \multirow[t]{3}{*}{ Nausea } & 0 & 38 & $95.0 \%$ \\
\hline & I & 1 & $2.5 \%$ \\
\hline & II & 1 & $2.5 \%$ \\
\hline \multirow[t]{2}{*}{ Vomiting } & 0 & 38 & $95.0 \%$ \\
\hline & I & 2 & $5.0 \%$ \\
\hline \multirow[t]{3}{*}{ Neutropenia } & 0 & 33 & $82.5 \%$ \\
\hline & I & 3 & $7.5 \%$ \\
\hline & II & 4 & $10.0 \%$ \\
\hline \multirow[t]{3}{*}{ Anemia } & 0 & 35 & $87.5 \%$ \\
\hline & I & 4 & $10.0 \%$ \\
\hline & II & 1 & $2.5 \%$ \\
\hline \multirow[t]{3}{*}{ Thrombocytopenia } & 0 & 38 & $95.0 \%$ \\
\hline & I & 1 & $2.5 \%$ \\
\hline & II & 1 & $2.5 \%$ \\
\hline
\end{tabular}

\section{DISCUSSION}

Diagnosis of mCRC became no longer meaning a rapid downhill course, as many patients live for years with what might be classified as a chronic disease ${ }^{23}$.

A big portion of patients in a palliative setting, will have no or only mild symptoms from their metastatic disease. In these patients, the main goals of therapy are extending PFS and maintaining the quality of life as long as possible. These patients will benefit from a careful long-term strategic planning of treatment sequences with mindful attention to treatment toxicities ${ }^{23}$. This mandates changes in therapy schedule, with treatment breaks or phases of less-intensive maintenance therapy interspersed with periods of more-intensive therapy to control tumor progression. This conceivably reduce the cumulative toxicities of therapy, potentially prevent the unplanned, premature discontinuation of therapy, preserve the ability to administer further phases of therapy, potentially maximize the time on therapy, reduce cost, and could increase quality of life for patients. One agent can be efficacious when used in various sequential phases of therapy ${ }^{24}$.

Maintenance capecitabine at low doses has been applied successfully in a number of small clinical trials. However, an effective dose of metronomic capecitabine has not been established; doses from 500 to $2,000 \mathrm{mg}$ daily can be found in the literature ${ }^{25}$.

In the current study, the median PFS was 34 weeks (7.9 months) which is comparable to that achieved with combination chemotherapy using oxaliplatin plus either 5-FU or capecitabine, as well as irinotecan plus 5-FU that expected to achieve a PFS of 7 to 9 months ${ }^{26-28}$.

Regarding the safety profile in the current study, no grade 4 toxicities were detected during the use of low dose capecitabine. Grade III was detected in only 3 patients $(7.5 \%)$ who developed grade III HFS. In contrast, the incidence of grade 3 HFS with standard dosing of capecitabine ranges from $16 \%$ to $44 \%$.

Toxicity was the cause of study termination in only 2 patients (5\%), while the third improved after treatment delay and dose reduction. This is also in contrast to standard-dose capecitabine, which is associated with a $13 \%$ discontinuance rate due to side effects ${ }^{31}$.

This toxicity profile was comparable to number of studies that evaluated the safety of use of low dose capecitabine in advanced GI malignancies ${ }^{3,8,12}$.

Results of the current study encourages the usage of the low dose capecitabine in minimally pretreated patients to maintain their response reserving more chemotherapy lines to subsequent stages, gaining time and better performance by reducing toxicity.

Ongoing clinical trial is testing maintenance chemotherapy with capecitabine versus control after best response with first line chemotherapy in advanced colorectal cancer, where the main endpoint is PFS $^{32}$. 
It is conceivable that the strength of biologics could well lie in being used as maintenance therapy after induction of response using conventional chemotherapy. This approach has shown success in the MACRO trial ${ }^{33}$. Also it is being tested in ongoing clinical trials ${ }^{34,35}$.

\section{CONCLUSION}

Maintenance low dose capecitabine may be effective in maintaining response in patients with $\mathrm{mCRC}$ patients with good tolerability.

This regimen should be further explored in larger prospective randomized controlled studies to demonstrate efficacy in mCRC as a maintenance therapy in minimally pretreated patients reserving more chemotherapy lines to subsequent stages. The usage of maintenance capecitabine could also be an option instead watchful waiting in the stable heavily pretreated mCRC cases.

\section{REFERENCES}

1. Gallagher DJ and Kemeny N. Metastatic colorectal cancer: From improved survival to potential cure. Oncology. 2010;78:237-248.

2. Pfeiffer P, Mortensen JP, Bjerregaard B, et al. Patient preference for oral or intravenous chemotherapy: a randomized cross-over trial comparing capecitabine and Nordic fluoro uracil/ leucovorin in patients with colorectal cancer. Eur J Cancer. 2006;42:2738-2743.

3. Petrioli R, Paolelli L, Marsili S, et al. FOLFOX-4 stop and go and capecitabine maintenance chemotherapy in the treatment of metastatic colorectal cancer. Oncology. 2006;70:345-350

4. Browder T, Butterfield CE, Kraling BM, et al. Antiangiogenic scheduling of chemotherapy improves efficacy against experimental drug-resistant cancer. Cancer Res. 2000;60:1878-1886.

5. Pietras K and Hanahan D. A multitargeted, metronomic, and maximum- tolerated dose "chemo-switch" regimen is antiangiogenic, producing objective responses and survival benefit in a mouse model of cancer. J Clin Oncol. 2005;23:939-952.

6. Kerbel RS and Kamen BA. The anti-angiogenic basis of metronomic chemotherapy. Nat Rev Cancer. 2004;4:423-436.

7. Loupakis F, Bocci G, Allegrini G, et al. Metronomic chemotherapy (MC) with irinotecan (CPT-11) in patients with diagnosis of metastatic colorectal cancer (MCRC): clinical and pharmacodynamic evaluation. J Clin Oncol, 2006 ASCO Annual Meeting Proceedings. 2006;24:13111.

8. Marshall J, Sun J and Norris C. Safety of chronic low dose capecitabine as maintenance therapy in GI cancers. 2007 Gastrointestinal Cancer Symposium. Abstract No: 166.

9. Li Y, Li J, Lu M, et al. Capecitabine Maintenance Therapy after First-Line Chemotherapy in Patients with Metastatic Colorectal Cancer. Chinese J Cancer Res. 2010;22: 181-185.
10. Li YH, Luo HY, Wang FH, et al. Phase II study of capecitabine plus oxaliplatin (XELOX) as first-line treatment and followed by maintenance of capecitabine in patients with metastatic colorectal cancer. J Cancer Res Clin Oncol. 2010;136:503-510.

11. Ogata Y, Akagi Y, Sasatomi T, et al. Phase I/II study of metronomic chemotherapy using S-1 and irinotecan in patients with advanced colorectal cancer (KSCOG CR01). J Clin Oncol. 2008;26: 2535.

12. Sun JF, $\mathrm{Wu} R R$, Norris $\mathrm{C}$ et al. Safety of Chronic Low-Dose Capecitabine as Maintenance Therapy in Gastrointestinal Cancers. Gastrointest Cancer Res. 2009;3:134:140.

13. Scalamogna R, Brugnatelli S, Tinelli $C$, et al. UFT as Maintenance Therapy in Patients with Advanced Colorectal Cancer Responsive to the FOLFOX4 Regimen. Oncology. 2007;72:267-273.

14. Steinbild S, Arends J, Medinger M, et al. Metronomic Antiangiogenic Therapy with Capecitabine and Celecoxib in Advanced Tumor Patients - Results of a Phase II Study. Onkologie. 2007;30:629-635.

15. Wu R, Sun J, Slack R, et al. Advanced Colorectal Cancer: Safety of Chronic Low-Dose Capecitabine as Maintenance Therapy in GI Cancers. International Society of GI Oncology- 2008 Meeting preview: 0823.

16. Hirsch BR and Zafar SY. Capecitabine in the management of colorectal cancer. Cancer Manag Res. 2011;3:79-89.

17. Van Cutsem E, Twelves C, Cassidy J, et al. Oral capecitabine compared with intravenous fluorouracil plus leucovorin in patients with metastatic colorectal cancer: results of a large phase III study. Xeloda Colorectal Cancer Study Group. J Clin Oncol. 2001;19:4097-4106.

18. Cassidy J, Twelves C, Van Cutsem E, et al. Capecitabine Colorectal Cancer Study Group. First line oral capecitabine therapy in metastatic colorectal cancer: a favorable safety profile compared with intravenous 5-fluorouracil/ leucovorin. Ann Oncol. 2002;13:566-575.

19. Oken MM, Creech RH, Tormey DC, et al. Toxicity and response criteria of the Eastern Cooperative Oncology Group. Am J Clin Oncol. 1982;5: 649-655.

20. Therasse P, Arbuck SG, Eisenhauer EA, et al. New guidelines to evaluate the response to treatment in solid tumors (RECIST Guidelines). J Natl Cancer Inst. 2000;92:205-216.

21. Casciato D and Territo M. Toxicities of chemotherapy: NCI- Common Toxicity Criteria version 3. In: Casciato D (ed). Manual of Clinical Oncology, $6^{\text {th }}$ Edition. 735:743. Phyladelphia: Lippincott Wiliams and Wilkins, 2009.

22. Lassere $Y$ and Hoff P. Management of hand-foot syndrome in patients treated with capecitabine (Xeloda). Eur J Oncol Nursing. 2004;8:S31-S40.

23. Goldberg RM, Rothenberg ML, Van Cutsem E, et al. The continuum of care: a paradigm for the management of metastatic colorectal cancer. Oncologist. 2007;12:38-50.

24. Grothey A. The Continuum of Care in Colorectal Cancer: Eliminating the Concept of Distinct Lines of Treatment. 2007 Educational Book, American Society of Clinical Oncology. 2007; 224-228.

25. Raftery L and O'Neil BH. Maintenance Capecitabine for High-Risk Gastrointestinal Tumors: Everything That's Old Is New Again. Gastrointest Cancer Res. 2009;3:163-164. 
26. Tournigand C, André T, Achille E, et al. FOLFIRI followed by FOLFOX6 or the reverse sequence in advanced colorectal cancer: a randomized GERCOR study. J Clin Oncol. 2004;22: 229-237.

27. Colucci G, Gebbia V, Paoletti G, et al. Phase III randomized trial of FOLFIRI versus FOLFOX4 in the treatment of advanced colorectal cancer: a multicenter study of the Gruppo Oncologico Dell'Italia Meridionale. J Clin Oncol. 2005;23:4866-4875.

28. Cassidy J, Clarke S, Díaz-Rubio E, et al. Randomized phase III study of capecitabine plus oxaliplatin compared with fluorouracil/folinic acid plus oxaliplatin as first-line therapy for metastatic colorectal cancer. J Clin Oncol. 2008;26: 2006-2012.

29. Van Cutsem E, Findlay M, Osterwalder B, et al. Capecitabine, an oral fluoropyrimidine carbamate with substantial activity in advanced colorectal cancer: results of a randomized phase II study. J Clin Oncol. 2000;18:1337-1345.

30. Van Cutsem E, Twelves C, Cassidy J, et al. Oral capecitabine compared with intravenous fluorouracil plus leucovorin in patients with metastatic colorectal cancer: results of a large phase III study. Xeloda Colorectal Cancer Study Group. J Clin Oncol. 2001;19:4097-4106.

31. Walko CM and Lindley C. Capecitabine: a review. Clin Ther. 2005;27:23-44.
32. Xeloda Vs Monitoring in Advanced Colorectal Cancer Responsive to Chemotherapy. National Cancer Institute (2011). Available at: http://clinicaltrials.gov/ct2/show/ study/NCT00189683

33. Tabernero J, Aranda E, Gómez A, et al. Phase III study of first-line XELOX plus bevacizumab (BEV) for 6 cycles followed by XELOX plus BEV or single agent (s/a) BEV as maintenance therapy in patients (pts) with metastatic colorectal cancer (mCRC): the MACRO trial (Spanish Cooperative Group for the Treatment of Digestive Tumors [TTD]). J Clin Oncol, ASCO Annual Meeting Proceedings. 2010;28:3501.

34. Study of Avastin (Bevacizumab) and Xeloda (Capecitabine) as Maintenance Treatment in Patients with Metastatic Colorectal Cancer. National Cancer Institute (2011). Available at: http://clinicaltrials.gov/ct2/show/ study/NCT00623805

35. Maintenance Treatment versus observation after induction in Advanced Colorectal Carcinoma (CAIRO3). National Cancer Institute (2011). Available at: http:// clinicaltrials.gov/ct2/show/study/NCT00442637.

36. Tournigand C, Scheithauer BSW, Andre CLT et al. mFOLFOX-bevacizumab or XELOX-bevacizumab then bevacizumab (B) alone or with erlotinib (E) in first-line treatment of patients with metastatic colorectal cancer (mCRC): Interim safety analysis of DREAM study. $\mathrm{J}$ Clin Oncol, ASCO Annual Meeting Proceedings. 2009;27:4077. 